\title{
Erste erfolgversprechende Ergebnisse der Kombination aus Strahlentherapie und Pembrolizumab beim metastasierten nichtkleinzelligen Lungenkarzinom
}

\author{
Markus Hecht ${ }^{1}$ - Udo S. Gaipl ${ }^{1} \cdot$ Rainer Fietkau ${ }^{1}$ \\ Online publiziert: 10. Februar 2020 \\ (c) Der/die Autor(en) 2020
}

Hintergrund und Ziele In der Therapie des metastasieren nichtkleinzelligen Lungenkarzinoms (NSCLC) werden neben der klassischen Chemotherapie und den Kinaseinhibitoren auch Immuncheckpoint-Inhibitoren, wie der PD-1Inhibitor Pembrolizumab, eingesetzt. In der oligometastasierten Situation liegen erste vielversprechende Ergebnisse zur lokal-ablativen Bestrahlung aller Metastasen ergänzend zur Chemotherapie vor [1]. In der Phase-II-Studie von Bauml et al. wird der Effekt von Pembrolizumab nach lokal ablativer Bestrahlung sämtlicher Metastasen beim NSCLC untersucht. Bei der Kombination von PD-1/PD-L1-Inhibitoren und Strahlentherapie gibt es aus der präklinischen Forschung gute Daten hinsichtlich der Induktion von lokalen und abskopalen Antitumor-Immunantworten (zusammengefasst in [2]). Die Studie von Theelen et al. geht noch einen Schritt weiter und untersucht die Bestrahlung nur einer einzelnen Metastase bei Patienten mit mehreren Metastasen. Es soll die Hypothese überprüft werden, ob durch die immunologischen Vorgänge in der bestrahlten Metastase die systemische Immunreaktion und damit das Therapieansprechen der Patienten verbessert werden kann.

Originalpublikation Bauml JM, Mick R, Ciunci C, Aggarwal C et al (2019) Pembrolizumab after completion of locally ablative therapy for oligometastatic non-small cell lung cancer: a phase 2 trial. JAMA Oncol. Jul 11. https://doi.org/10.1001/jamaoncol. 2019.1449.

Theelen WSME, Peulen HMU, Lalezari F, van der Noort V (2019) Effect of pembrolizumab after stereotactic body radiotherapy vs pembrolizumab alone on tumor response in patients with advanced non-small cell lung cancer: results of the PEMBRO-RT phase 2 randomized clinical trial. JAMA Oncol. Jul 11. https://doi.org/10.1001/jamaoncol.2019.1478.

PD Dr. med. Markus Hecht

markus.hecht@uk-erlangen.de

1 Universitäts-Strahlenklinik Erlangen,

Friedrich-Alexander-Universität Erlangen-Nürnberg,

Universitätsstraße 27, 91054 Erlangen, Deutschland
Patienten und Methoden (Bauml et al.) In diese einarmige Phase-II-Studie konnten Patienten mit bis zu 4 Metastasen eines NSCLC unabhängig vom PD-L1-Status eingeschlossen werden. Die Studie erfolgte monozentrisch. Vor Studieneinschluss mussten alle Metastasen lokal ablativ behandelt worden sein. Als lokale Verfahren waren Operation, Radiochemotherapie, stereotaktische Bestrahlung und interventionelle Verfahren erlaubt. Im Anschluss erfolgte die Therapie mit Pembrolizumab in der üblichen Dosierung (200 mg Absolutdosis, q21d). Primärer Endpunkt der Studie war das progressionsfreie Überleben (PFS), gemessen zum einen nach Beginn der lokal ablativen Therapie und zum anderen nach Beginn der Therapie mit Pembrolizumab. Das PFS der Studienpatienten sollte mit einer historischen Kontrollkohorte verglichen werden, die eine alleinige ablative Bestrahlung erhielt. Lebensqualität wurde mit dem FACTL-Fragebogen bestimmt.

Ergebnisse (Bauml et al.) Zwischen Februar 2015 und September 2017 wurden 51 Patienten in die Studie eingeschlossen, von denen 45 mit Pembrolizumab behandelt wurden. Die Analysen der Studie beziehen sich nur auf die 45 Patienten, die Pembrolizumab erhielten. In dieser Kohorte betrug das mediane PFS seit Beginn der ablativen Verfahren 19,1 Monate. Verglichen mit der historischen Kontrolle mit alleiniger ablativer Bestrahlung war dies ein signifikanter Vorteil (19,1 Monate vs. 6,6 Monate, 95\% CI 9,4-28,7 Monate, $p<0,005)$. Das Gesamtüberleben betrug nach einem Jahr 90,9\% und nach zwei Jahren 77,5\%. Die Kombination der beiden Therapien führte zu keinen relevant verstärkten Nebenwirkungen und zu keiner schlechteren Lebensqualität.

Schlussfolgerung von Bauml et al. Pembrolizumab scheint das PFS nach lokal ablativer Therapie beim NSCLC zu verbessern, ohne die Lebensqualität einzuschränken. 
Patienten und Methoden (Theelen et al.) In der der multizentrischen PEMBRO-RT-Studie wurden Patienten mit metastasiertem Bronchialkarzinom nach Progress auf Erstlinien-Chemotherapie behandelt. Er erfolgte eine Randomisation auf entweder Pembrolizumab-Monotherapie (200 mg Absolutdosis, q21d) oder die Kombination aus Pembrolizumab und stereotaktischer Bestrahlung einer einzelnen Metastase mit $3 \times 8 \mathrm{~Gy}$. Es musste mindestens eine weitere unbestrahlte Metastase zur Beurteilung des Therapieansprechens nach RECIST-Kriterien verbleiben. Die Immuntherapie musste innerhalb von 7 Tagen nach Abschluss der stereotaktischen Bestrahlung eingeleitet werden. Primärer Endpunkt war die Ansprechrate (,overall response rate“, ORR). Studienhypothese war die Verbesserung der ORR nach 12 Wochen von 20 auf $50 \%$ mit $p<0,10$.

Ergebnisse (Theelen et al.) Insgesamt wurden 76 Patienten randomisiert, im Kontrollarm 40 Patienten und im Prüfarm (Kombination mit Bestrahlung) 36 Patienten. Bei den meisten Patienten wurden Lungen- oder Lymphknotenmetastasen bestrahlt. Der primäre Endpunkt ORR betrug $18 \%$ im Kontrollarm und $36 \%$ im Prüfarm $(p=0,07)$. Das mediane progressionsfreie Überleben lag im Kontrollarm bei 1,9 Monaten und im Prüfarm bei 6,6 Monaten (HazardRatio 0,$71 ; 95 \%$ CI 0,42-1,18; $p=0,19)$. Das mediane Gesamtüberleben lag im Kontrollarm bei 7,6 Monaten und im Prüfarm bei 15,9 Monaten (Hazard-Ratio 0,66; $95 \%$ CI $0,37-1,18 ; \beta=0,16)$. Interessanterweise profitierten insbesondere Patienten mit PD-L1-negativen Tumoren von der Kombination mit Strahlentherapie. Die Nebenwirkungsrate war im Prüfarm nicht erhöht.

Schlussfolgerung von Theelen et al. Obwohl die ORR verdoppelt wurde, wurde der primäre Endpunkt der Studie nicht erreicht. Die Verbesserung durch Kombination der Bestrahlung trat im Wesentlichen in der PD-L1-negativen Subgruppe auf. Die Ergebnisse legen dennoch die Durchführung einer größeren Studie nahe.

\section{Kommentar}

Pembrolizumab ist zur Behandlung des metastasierten Bronchialkarzinoms zugelassen, wobei die Zulassung an das Vorhandensein des prädiktiven Parameters PD-L1 gebunden ist. Bei Tumoren mit Expression des prädiktiven Markers PD-L1 auf mindestens 1\% der Tumorzellen (TPSScore $\geq 1 \%$ ) ist Pembrolizumab in der Zweitlinientherapie zugelassen. Bei Expression von PD-L1 auf mindestens 50\% der Tumorzellen (TPS-Score $\geq 50 \%$ ), kann Pembrolizumab bereits in der Erstlinientherapie eingesetzt werden. So steht Patienten im metastasierten Stadium neben der klassischen
Chemotherapie und den Kinase-Inhibitoren nun mit den PD-1-Inhibitoren eine neue Therapieoption zur Verfügung.

Bei Patienten mit Therapieansprechen auf die initiale Chemotherapie und Oligometastasierung, in dieser Studie definiert mit bis zu 3 Metastasen, konnte kürzlich auch eine signifikante Verbesserung des Überlebens durch konsequente Lokaltherapie gezeigt werden [1]. Bisher gab es noch keine Daten zur konsequenten Lokaltherapie in Kombination mit Immuntherapie. In der Studie von Bauml et al. erhielten Patienten mit Oligometastasierung neben der Lokaltherapie aller Läsionen eine Immuntherapie mit Pembrolizumab. Leider werden in der Publikation nur die gesamten Vortherapien der Patienten angegeben und nicht die aktuell angewendeten Lokaltherapie-Verfahren, sodass nicht unterschieden werden kann, wie viele Patienten strahlentherapeutisch und wie viele chirurgisch lokal behandelt wurden. Mit der Kombination aus Lokaltherapie und Pembrolizumab wurde aber ein beachtliches PFS von 19,1 Monaten erreicht, das der historischen Kontrolle mit alleiniger Bestrahlung weit überlegen war. Dies ist nicht verwunderlich, da die Wirksamkeit von Pembrolizumab in diesem Patientenkollektiv bereits vielfach belegt wurde [3]. Beachtlich ist jedoch die Überlebensrate von 90,9\% nach einem Jahr und 77,5\% nach 2 Jahren. In der Zweitlinientherapie mit Pembrolizumab lagen die Überlebensraten üblicherweise bei etwa $50 \%$ nach einem Jahr [3]. Auch wenn bisher nur Daten aus Phase-II-Studien vorliegen, scheint eine intensive Lokaltherapie bei Patienten mit Oligometastasierung nicht nur in Kombination mit Chemotherapie, sondern auch in Kombination mit Immuntherapie ein hoch effektives Therapieverfahren zu sein.

Hinsichtlich Strahlentherapie und PD-1-Inhibitoren gibt es zahlreiche präklinische Daten, dass sich beide Verfahren nicht nur wie in obiger Studie gut kombinieren lassen, sondern auch gegenseitig in ihrer Wirkung verstärken. Es ist lange bekannt, dass Strahlentherapie neben den klassischen Zelltod über die Akkumulation von DNA-Schäden auch den Tumorzell-Phänotyp und das Tumormikromilieu verändert [2]. So konnte in präklinischen Experimenten bei Kombination von Strahlentherapie und PD-1/PD-L1-Inhibitoren neben der lokalen Wirkung auch eine Verstärkung der systemischen Wirkung erreicht werden.

In der Studie von Theelen et al. wurde untersucht, ob dieser Effekt auch bei Patienten genutzt werden kann, um die Effektivität der Immuntherapie zu steigern. In dieser Studie wurde eine Metastase mit $3 \times 8 \mathrm{~Gy}$ bestrahlt und innerhalb von 7 Tagen die Therapie mit Pembrolizumab eingeleitet. $\mathrm{Ob}$ eine andere Sequenz der Behandlungen oder eine andere Bestrahlungsdosis möglicherweise besser zur Induktion immunologischer Effekte geeignet ist, konnte bisher nicht geklärt werden. Aktuell wird diskutiert, dass insbesondere die Bestrahlung von mehreren Metastasen ein günstiges im- 
munologisches Milieu schafft [4]. In der Studie konnte die Ansprechrate auf Pembrolizumab von 18 auf $36 \%$ verdoppelt werden, jedoch nicht statistisch signifikant $(p=0,07)$. Die fehlende statistische Signifikanz ist bei der zugrundeliegenden Fallzahlkalkulation mit der Annahme einer Verbesserung von 20 auf $50 \%$ kalkuliert, mit $p<0,10$ nicht verwunderlich. Mit der so berechneten Fallzahl von $74 \mathrm{~Pa}-$ tienten (76 Patienten wurden randomisiert) sind kaum signifikante Werte zu erwarten. Dennoch kann das Ergebnis dieser Phase-II-Studie als deutliches Signal gewertet werden, dass eine lokale Bestrahlung auch systemische Effekte bewirken kann, die die Wirksamkeit einer Immuntherapie verstärken. Ein weiteres, sehr interessantes Ergebnis ist die Beobachtung, dass vor allem Patienten mit PD-L1-negativen Tumoren von einer zusätzlichen Bestrahlung profitieren. Es ist bekannt, dass PD-L1 nach Radiochemotherapie auf Tumoren in Patienten hochreguliert wird [5]. Da PDL1 der wichtigste prädiktive Parameter für die Therapie mit PD-1-Inhibitoren ist, ist es nicht verwunderlich, dass von der Bestrahlung insbesondere Patienten mit primär PD-L1negativen Tumoren profitieren. Auch gilt es zu bedenken, dass zum Testen des PD-L1-Status Biopsien nach initialer Bestrahlung genommen werden sollten, da diese stark die Expression von PD-L1 beeinflussen kann [6].

Trotz dieser beeindruckenden Daten zur systemischen Wirkung der lokalen Bestrahlung muss beachtet werden, dass beim diesjährigen ASCO eine fast identische PhaseII-Studie bei Kopf-Hals-Tumoren diskutiert wurde, die keine systemische Wirkung der lokalen Strahlentherapie nachweisen konnte [7]. In zehn deutschen Studienzentren wird derzeit im Rahmen der IMPORTANCE-Studie ein ähnliches Konzept - Kombination von fraktionierter Bestrahlung und Pembrolizumab - in der Erstlinientherapie von metastasierten Kopf-Hals-Tumoren untersucht. Auf das Ergebnis darf man gespannt sein.

\section{Fazit}

Bei Patienten mit Oligometastasierung eines NSCLC scheint die intensive Lokaltherapie nicht nur in Kombination mit Chemotherapie, sondern auch mit Immuntherapie sehr gute Ergebnisse zu erzielen.

Eine erste Phase-II-Studie liefert Hinweise auf systemische Effekte einer lokalen Strahlentherapie, die das Therapieansprechen auf PD-1-Inhibitoren verbessern könnten.

Markus Hecht, Udo S. Gaipl und Rainer Fietkau, Erlangen

Funding Open Access funding provided by Projekt DEAL.

Interessenkonflikt $\mathrm{M}$. Hecht hat an Advisory Boards von MSD, Merck Serono und BMS teilgenommen, Forschungsförderung von
MSD, AstraZeneca und Novartis und Reiseunterstützung von Merck Serono, MSD und Teva erhalten. U.S. Gaipl hat an Advisory Boards von AstraZeneca und BMS teilgenommen und erhält Forschungsförderung von MSD und AstraZeneca. R. Fietkau hat an Advisory Boards von Merck Serono, AstraZeneca, MSD, Novocure, Brainlab, Fresenius Kabi und BMS teilgenommen und Forschungsförderung von Merck Serono, AstraZeneca, MSD, Novocure, Brainlab, Fresenius Kabi, Sennewald und BMS erhalten.

Open Access Dieser Artikel wird unter der Creative Commons Namensnennung 4.0 International Lizenz veröffentlicht, welche die Nutzung, Vervielfältigung, Bearbeitung, Verbreitung und Wiedergabe in jeglichem Medium und Format erlaubt, sofern Sie den/die ursprünglichen Autor(en) und die Quelle ordnungsgemäß nennen, einen Link zur Creative Commons Lizenz beifügen und angeben, ob Änderungen vorgenommen wurden.

Die in diesem Artikel enthaltenen Bilder und sonstiges Drittmaterial unterliegen ebenfalls der genannten Creative Commons Lizenz, sofern sich aus der Abbildungslegende nichts anderes ergibt. Sofern das betreffende Material nicht unter der genannten Creative Commons Lizenz steht und die betreffende Handlung nicht nach gesetzlichen Vorschriften erlaubt ist, ist für die oben aufgeführten Weiterverwendungen des Materials die Einwilligung des jeweiligen Rechteinhabers einzuholen.

Weitere Details zur Lizenz entnehmen Sie bitte der Lizenzinformation auf http://creativecommons.org/licenses/by/4.0/deed.de.

\section{Literatur}

1. Gomez DR, Tang C, Zhang J, Blumenschein GR Jr., Hernandez M, Lee JJ, Ye R, Palma DA, Louie AV, Camidge DR, Doebele RC, Skoulidis F, Gaspar LE, Welsh JW, Gibbons DL, Karam JA, Kavanagh BD, Tsao AS, Sepesi B, Swisher SG, Heymach JV (2019) Local Consolidative therapy vs. Maintenance therapy or observation for patients with Oligometastatic non-small-cell lung cancer: long-term results of a multi-institutional, phase II, randomized study. J Clin Oncol 37(18):1558-1565. https://doi.org/10.1200/ JCO.19.00201

2. Derer A, Frey B, Fietkau R, Gaipl US (2015) Immune-modulating properties of ionizing radiation: rationale for the treatment of cancer by combination radiotherapy and immune checkpoint inhibitors. Cancer Immunol Immunother. https://doi.org/10.1007/ s00262-015-1771-8

3. Herbst RS, Baas P, Kim DW, Felip E, Perez-Gracia JL, Han JY, Molina J, Kim JH, Arvis CD, Ahn MJ, Majem M, Fidler MJ, de Castro G Jr., Garrido M, Lubiniecki GM, Shentu Y, Im E, Dolled-Filhart M, Garon EB (2016) Pembrolizumab versus docetaxel for previously treated, PD-L1-positive, advanced non-smallcell lung cancer (KEYNOTE-010): a randomised controlled trial. Lancet 387(10027):1540-1550. https://doi.org/10.1016/S01406736(15)01281-7

4. Brooks ED, Chang JY (2019) Time to abandon single-site irradiation for inducing abscopal effects. Nat Rev Clin Oncol 16(2):123-135. https://doi.org/10.1038/s41571-018-0119-7

5. Hecht M, Buttner-Herold M, Erlenbach-Wunsch K, Haderlein M, Croner R, Grutzmann R, Hartmann A, Fietkau R, Distel LV (2016) PD-L1 is upregulated by radiochemotherapy in rectal adenocarcinoma patients and associated with a favourable prognosis. Eur J Cancer 65:52-60. https://doi.org/10.1016/j.ejca.2016.06.015

6. Lim YJ, Koh J, Kim S, Jeon SR, Chie EK, Kim K, Kang GH, Han SW, Kim TY, Jeong SY, Park KJ, Wu HG (2017) Chemoradiation-induced alteration of programmed death-Ligand 1 and CD8(+) tumor-infiltrating lymphocytes identified patients with poor prognosis in rectal cancer: a matched comparison analysis. Int J Radiat 
Oncol Biol Phys 99(5):1216-1224. https://doi.org/10.1016/j.ijrobp. 2017.07.004

7. McBride SM, Sherman EJ, Tsai CJ, Baxi SS, Aghalar J, Eng J, Zhi WI, McFarland DC, Michel LS, Spielsinger D, Zhang Z, Flynn J, Dunn L, Ho AL, Riaz N, Pfister DG, Lee NY (2018)
A phase II randomized trial of nivolumab with stereotactic body radiotherapy (SBRT) versus nivolumab alone in metastatic (M1) head and neck squamous cell carcinoma (HNSCC). J Clin Oncol 36(15_suppl):6009 